\title{
Prevalência de hipertensão arterial em crianças e adolescentes: Revisão integrativa*
}

\section{Hypertension Prevalence in Children and Adolescents: integrative review*}

\author{
Viviane Silva de Jesus' - Samylla Maira Costa Siqueira ${ }^{2}$ Elane Nayara Batista dos Santos ${ }^{3}$. \\ Juliana Pedra de Oliveira Muniz ${ }^{4} \cdot$ Ridalva Dias Martins Felzemburgh ${ }^{5} \cdot$ Climene Laura de Camargo $^{6}$
}

\begin{abstract}
RESUMO
Objetiva-se identificar as publicações sobre prevalência de hipertensão arterial em crianças e adolescentes e os manuais referenciados nos estudos. Trata-se de uma revisão integrativa, com busca realizada nas bases de dados Literatura LatinoAmericana e do Caribe em Ciências da Saúde (LILACS), Medical Literature Analysis and Retrieval Sistem on-line (Medline), Base de Dados em Enfermagem (BDENF) e, na biblioteca eletrônica Scientific Electronic Library Online (SciELO) com associação dos descritores "hipertensão arterial","criança" "adolescente". Os artigos foram avaliados quantitativamente e, a organização dos dados foi realizada por similaridade. Foram inclusos 54 estudos, 94,4\% (51) utilizaram o delineamento transversal, com prevalência entre 1,5\% a 70,5\%. 0 "The Fourth Report on the Diagnosis, Evaluation, and Treatment of High Blood Pressure in Children and Adolescents" (2004), foi o manual mais utilizado, abrangendo 46,3\% (25). Identificouse um cenário crescente e diferenciado da prevalência de hipertensão arterial na infância e adolescência, o que leva à aplicação de recomendações descritas em manuais, a fim de facilitar o acompanhamento, tratamento, bem como viabilizar o diagnóstico precoce da doença.
\end{abstract}

Palavras-chave: Hipertensão Arterial; Criança; Adolescente.

\section{ABSTRACT}

The aim is to identify publications on the hypertension prevalence in children and adolescents and manuals referenced in the studies. This is an integrative review, with search performed in the databases Literature Latin American and Caribbean Health Sciences (LILACS), Medical Literature Analysis and Retrieval System Online (Medline), Nursing Database (BDENF) and in Scientific Electronic Library Online (SciELO), with the association of descriptors "high blood pressure", "child" and "adolescent". Articles were evaluated quantitatively and the organization of data was performed by similarity. 54 studies were included, $94.4 \%$ (51) used the cross-sectional design, with prevalence from $1.5 \%$ to $70.5 \%$. The "The Fourth Report on the Diagnosis, Evaluation, and Treatment of High Blood Pressure in Children and Adolescents" (2004), was the most widely used manual covering $46.3 \%$ (25). Were identified a growing and differentiated scene of the prevalence of hypertension in childhood and adolescence, which leads to the implementation of recommendations described in manuals in order to facilitate the monitoring, treatment, and facilitate early diagnosis of the disease.

Keywords: Hypertension; Child; Adolescent.

NOTA

${ }^{1}$ Enfermeira. Mestre em Enfermagem. Doutoranda em Saúde pública pelo Instituto de Saúde Coletiva da Universidade Federal da Bahia. Docente pela Faculdade Adventista da Bahia, Cachoeira-BA.

${ }^{2}$ Enfermeira. Mestre e Doutoranda em Enfermagem pelo Programa de Pós-Graduação em Enfermagem e Saúde da Universidade Federal da Bahia, Salvador, Bahia. Docente do Centro Universitário Jorge Amado, Salvador-BA, Brasil.

${ }^{3}$ Enfermeira graduada pela Escola de Enfermagem da Universidade Federal da Bahia, Salvador, Bahia.

${ }^{4}$ Enfermeira. Mestranda em Enfermagem do Programa de Pós-Graduação em Enfermagem da Universidade Federal da Bahia, Salvador, Bahia.

${ }^{5}$ Enfermeira. Doutora. Docente do Programa de Pós-Graduação em Enfermagem da Universidade Federal da Bahia, Salvador, Bahia.

${ }^{6}$ Enfermeira. Doutora. Docente do Programa de Pós-Graduação em Enfermagem da Universidade Federal da Bahia, Salvador, Bahia.

*Autoria de Viviane Silva de Jesus. Título: Fatores Relacionados à Elevação de Níveis Pressóricos em Crianças Quilombolas. Categoria: Dissertação de Mestrado. Programa de Pós-Graduação em Enfermagem da Universidade Federal da Bahia, Salvador, Bahia. Apresentado em 2015. Declaramos não haver conflitos de interesses e, a referida dissertação foi um recorte de um projeto maior financiado pela Fundação de Amparo à Pesquisa (FAPESB) sob edital $n^{\circ}$ edital $20 / 2013$. 


\section{INTRODUÇÃO}

A Hipertensão Arterial (HA) é uma doença multifatorial, configurada pelo aumento dos níveis tensionais dos vasos sanguíneos levando a níveis elevados da pressão arterial ${ }^{1-2}$. Doença de caráter silencioso e progressivo que pode gerar sequelas transitórias e permanentes, gerando ônus aos cofres públicos procedente da interrupção da vida produtiva ${ }^{3}$.

Reconhecida como um importante fator de risco para as doenças cardiovasculares, a HA assume destaque como uma preocupação de saúde pública mundial devido à sua alta prevalência acometendo $25 \%$ da população mundial $^{3}$. No Brasil, a HA também tem sido motivo de preocupação e desafio para profissionais e gestores de saúde, considerando que cerca de 22 a $43,9 \%$ da população adulta é hipertensa. A prevalência de hipertensão arterial entre crianças e adolescentes tem se mostrado crescente e cada vez mais presente nessas fases de vida, corroborando com a afirmativa de que um adulto hipertenso pode ter apresentado níveis elevados de pressão arterial na infância, acentuando-se na adolescência4.

Estudos internacionais e nacionais constataram a crescente prevalência da hipertensão arterial na infância e adolescência ao apresentar resultados que variam entre $5,2 \%$ a $52,4 \%{ }^{4-6}$. Essa larga diferença entre os achados pode transcorrer das distintas metodologias empregadas, em especial no que diz respeito aos pontos de corte adotados e ao número de aferições da pressão realizada durante $o$ atendimento. Tal situação impetra a necessidade de padronização para mensuração e avaliação da pressão arterial em todas as faixas etárias, especialmente na infância e adolescência. Estes manuais são consensos, relatórios e diretrizes que norteiam a técnica de aferição da pressão arterial, trazendo informações sobre diagnóstico, avaliação e tratamento da doença ${ }^{6}$.

O interesse em tornar a aferição da pressão a mais fidedigna possível, levou especialistas europeus e americanos a elaborarem regulamentos sobre técnicas. Tendo sua primeira publicação em 1939, intitulada "Recomendações da American Heart Association", abordando assuntos sobre a padronização do instrumental, posição do cliente e método de adaptação do manguito ${ }^{7}$.

Nos Estados Unidos e Europa, foram realizadas diversas revisões nas recomendações publicadas em $1939^{7}$. Contudo, foi a partir da década de 70 que as discussões sobre medidas de pressão arterial em crianças e adolescentes tomaram impulso, também em outras partes do mundo, passando por diversas modificações até a época vigente ${ }^{8}$.

Observa-se que o uso de normas para a avaliação da $\mathrm{PA}$, tem sido cada vez mais efetivo para a identificação da hipertensão em idades mais precoces, e sua importância tem se instituído uma vez que auxilia na prevenção primária das doenças cardiovasculares e estabelecimento da pressão alta na fase adulta ${ }^{6-9}$.

Embora a mensuração da pressão arterial seja, em todo o mundo, uma das práticas entre os profissionais de saúde e os manuais para avaliação da pressão arterial apresentem descrições detalhadas de técnicas para que se tenha um resultado fidedigno e seguro, muitos profissionais não têm conhecimento satisfatório acerca das normas, das bases teorias e prática deste procedimento ${ }^{10}$.

Considerando que os profissionais de enfermagem também são os que mais executam a aferição da pressão arterial, é de suma importância o aprimoramento, para realização deste procedimento ${ }^{10}$. Com isso, torna-se relevante trazer à luz os manuais mais utilizados para nortear as investigações sobre hipertensão arterial em crianças e adolescentes.

Assim, objetivou-se identificar as publicações sobre prevalência de hipertensão arterial em crianças e adolescentes e os manuais referenciados nos estudos.

\section{MÉTODO}

Trata-se de uma revisão integrativa da literatura,visando colaborar com o desenvolvimento do conhecimento a respeito do tema, a partir da avaliação e sintetização de outros resultados disponíveis ${ }^{11}$.

$O$ referido estudo foi elaborado em um processo de seis fases: elaboração da pergunta norteadora, definição dos critérios de inclusão, definição das informações a serem aproveitadas e elaboração de um instrumento, análise das informações registradas, interpretação e discussão dos dados, e por último, a apresentação da revisão integrativa ${ }^{12}$.

Foram incluídos os artigos originais que investigaram a prevalência de hipertensão arterial em crianças e adolescentes, informavam utilizar algum tipo de manual de orientação para investigação de hipertensão arterial, disponíveis gratuitamente e na íntegra, nos idiomas português, inglês e espanhol, publicados no período de 2004 a 2014, selecionados a partir do título, resumo e leitura na íntegra dos artigos. Para as publicações replicadas nas bases de dados, optou-se por uma única análise.

A pesquisa foi norteada pelo seguinte questionamento: Qual a prevalência de HA em criança e adolescentes retratadas nos artigos científicos e quais os manuais utilizados nesses estudos?

O levantamento dos artigos foi realizado nas bases de dados Literatura Latino-Americana e do Caribe em Ciências da Saúde (LILACS) e Medical Literature Analysis and Retrieval Sistem on-line (Medline), Base de Dados em Enfermagem (BDENF) e, na biblioteca eletrônica Scientific Electronic Library Online (SciELO).

A coleta de dados foi realizada nos meses de fevereiro a julho de 2015 por duas pesquisadoras e duas estudantes de enfermagem devidamente instruídas. Foram utilizados os descritores "hipertensão arterial", "criança" e "adolescente" em três etapas: primeira, foi realizada a busca pelos descritores hipertensão e criança; em seguida, com os descritores hipertensão e adolescentes; e por último, os três descritores, hipertensão, criança e adolescente, 
empregando como conector o operador booleno "AND" em todas as fases.

Foram identificados 39.229 estudos nas bases de dados avaliadas, dos quais: 6.230 na BEDENF, 1.022 na LILACS, 234 na SciELO e 31.743 na MEDLINE. Foi realizada a leitura dos títulos destes e depois excluídos todos os artigos que tratavam de outros tipos de hipertensão, resultando 3.571 produções (45, 575, 175 e 2776 nas bases acima citadas respectivamente). Posteriormente, os 3.571 artigos foram reunidos em uma só pasta, e realizada a leitura flutuante dos resumos culminando na seleção de 128 produções. Após a primeira leitura na íntegra, 9 artigos foram excluídos por duplicação e 119 elegíveis para o próximo passo. Na segunda leitura, identificou-se 65 artigos que não atendiam aos objetivos propostos, totalizando assim 54 estudos a serem inclusos para a revisão integrativa, sendo 22 em língua inglesa, 24 em português e 8 no idioma espanhol, como verifica-se na figura 1.

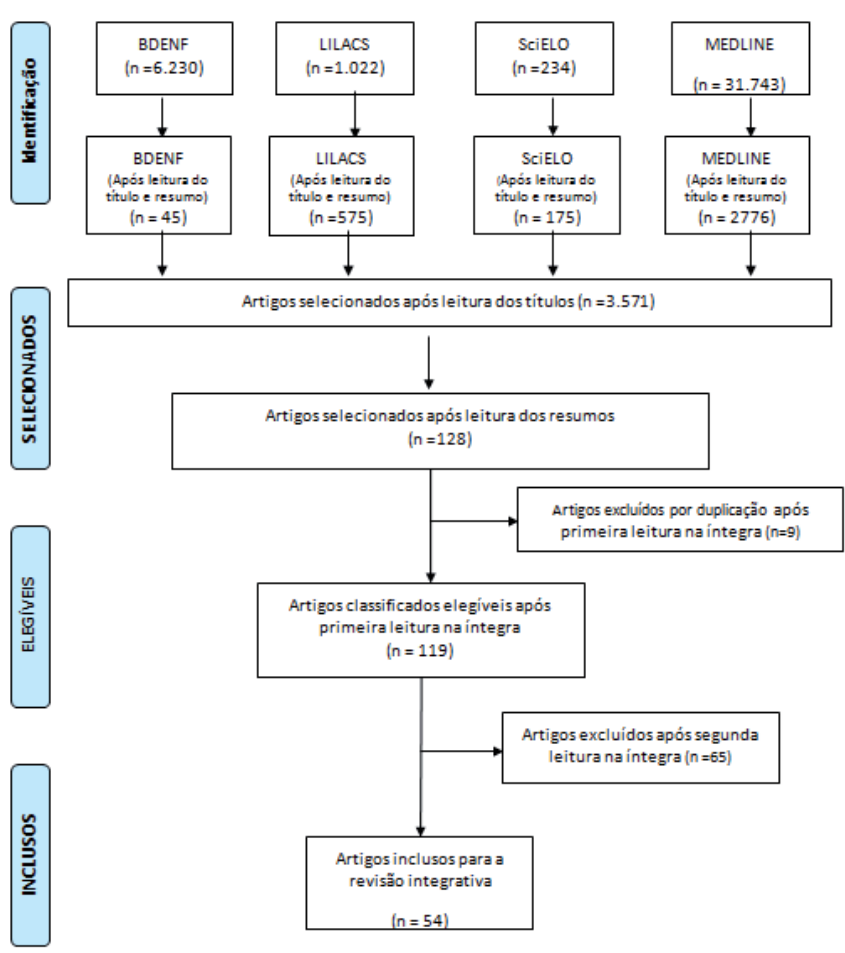

Figura 1 - Diagrama da seleção dos artigos por grupo de base de dados e total inclusos para revisão integrativa, Salvador-BA, 2015. *Modelo baseado no diagrama do Grupo PRISMA (2009).

Fonte: dados da pesquisa.

A fim de possibilitar melhor organização, utilizou-se um fichamento, construído pela autora, para tratamento dos estudos selecionados. Em seguida, os artigos passaram por uma terceira leitura na íntegra, com intuito de analisalos de maneira interpretativa, baseado no questionamento levantado e objetivo do estudo. Os resultados foram dispostos em quadros e tabela contendo a síntese dos estudos.

Para organização das informações contidas nos 54 artigos elegíveis, utilizou-se um instrumento, construído pela autora, com intuito de analisar as produções de maneira interpretativa, baseado no questionamento levantado e objetivo proposto. Os resultados foram dispostos em quadros e tabela contendo a síntese dos estudos. A seguir, segue diagrama da seleção dos artigos.

\section{RESULTADOS E DISCUSSÃO}

Dos 3.571 artigos identificados, foram selecionados 128 , dos quais 54 foram incluídos para análise integrativa. O quadro 1 apresenta características dos estudos conforme primeiro autor, formação, idioma, ano, país e periódicos de publicação que integram a presente revisão.

Em relação às profissões dos autores dos referidos estudos identificaram-se: médicos (44,4\%), Nutricionistas (20,4\%), Educadores Físicos (14,8\%) e Enfermeiros (11,1\%). A despeito da afirmativa de que em todo o mundo o Enfermeiro e o Médico são os profissionais que mais executam a técnica de aferição de pressão arterial ${ }^{13-14}$, os estudos analisados demonstram que os Enfermeiros ainda se mostram tímidos quanto às pesquisas abordando hipertensão em crianças e adolescentes.

Os países e idiomas em que mais foram publicados artigos referentes a hipertensão em crianças e adolescentes foram: Brasil, 38 estudos (22 em português e 16 em inglês); Argentina (dois em espanhol e um em inglês) e Chile (três em espanhol), três estudos ambos; Estados Unidos, dois estudos (inglês); Tobago (inglês), Venezuela (espanhol), Índia (inglês); África do Sul (português), Cuba (espanhol), Irã (português), Paraguai (espanhol) e China (inglês), com um estudo cada. Os estudos sobre hipertensão arterial em crianças e adolescentes no Brasil tem se mostrado cada vez mais expressivo, em consonância com o panorama epidemiológico apresentado no país 4 .

Quanto ao ano de publicação, constatou-se que quatro (9,3\%) foram publicados em 2004, um (1,9\%) em 2005, quatro $(7,4 \%)$ em 2006 , três $(5,6 \%)$ em 2007 , dois $(3,7 \%)$ em 2008 , sete $(13 \%)$ em 2009 , nove $(16,7 \%)$ em 2010 , sete (13\%) em 2011, cinco (9,3\%) em 2012, seis (11,1\%) em 2013 e quatro $(7,4 \%)$ em 2014 . Verifica-se que as publicações relacionadas à hipertensão arterial em crianças e adolescentes ascendem de forma contínua, principalmente no Brasil, corroborando com outros estudos ${ }^{4,15}$.

A maior proporção de publicação deu-se nos periódicos Jornal de Pediatria com 10 (18,5\%), seguidos das Revistas de Cardiologia com nove (16,7\%) e de Enfermagem com quatro $(7,4)$ estudos cada.

No quadro 2 a seguir, será descrita a trajetória metodológica dos estudos incluídos nesta:

Entre os estudos analisados 51 (94,4\%) apresentaram o delineamento metodológico transversal, seguidos de 1 caso-controle, 1 longitudinal e 1 coorte, com variação amostral entre 43 e 7.440 .

Em relação à prevalência de hipertensão arterial em crianças e adolescentes, os estudos analisados 
Quadro 1. Características gerais segundo primeiro autor, formação, idioma, ano, país e periódicos de publicação dos estudos sobre hipertensão em crianças e adolescentes publicados entre 2004 e 2014. Salvador, BA. 2015.

\begin{tabular}{|c|c|c|c|c|c|c|}
\hline $\mathrm{N}^{\circ}$ & Autor & Formação & Idioma & Ano & País & Periódico \\
\hline 1 & Elias MC et al & Nutrição & $\mathrm{Pt}$ & 2004 & Brasil & Arq. Bras. Cardiol. \\
\hline 2 & Garcia FD et al & Medicina & $\mathrm{Pt}$ & 2004 & Brasil & J. Pediatr. \\
\hline 3 & Oliveira AMA et al & Medicina & $\mathrm{Pt}$ & 2004 & Brasil & Arq. Bras. Endocrinol. Metab. \\
\hline 4 & Moura AA & Medicina & $\mathrm{Pt}$ & 2004 & Brasil & J. Pediatr. \\
\hline 5 & Sorof JM & Medicina & Ing & 2004 & EUA & The Journal of Pediatrics \\
\hline 6 & Silva MAM & Medicina & $\mathrm{Pt}$ & 2005 & Brasil & Arq. Bras. Cardiol. \\
\hline 7 & Nichols S, Cadogan F & Não identificado & Ing & 2006 & Tobago & West Indian Med J \\
\hline 8 & Rosa MLG & Medicina & $\mathrm{Pt}$ & 2006 & Brasil & Arq. Bras. Cardiol. \\
\hline 9 & Croix B, Feig DI & Enfermagem & Ing & 2006 & EUA & Pediatr. Nephrol. \\
\hline 10 & Monego ET, Jardim PCBV & Nutrição & $\mathrm{Pt}$ & 2006 & Brasil & Arq. Bras. Cardiol. \\
\hline 11 & Rosa MLG et al & Medicina & Ing & 2007 & Brasil & Arq. Bras. Cardiol. \\
\hline 12 & Borges LMP et al & Medicina & Ing & 2007 & Brasil & Rev. Saúde Pública \\
\hline 13 & Nogueira PCK et al & Medicina & Ing & 2007 & Brasil & Rev. Assoc. Med. Bras. \\
\hline 14 & Araújo TL et al & Enfermagem & $\mathrm{Pt}$ & 2008 & Brasil & Rev. esc. Enferm. USP \\
\hline 15 & Silva KS, e Lopes AS & Educação Física & Ing & 2008 & Brasil & Arq. Bras. Cardiol. \\
\hline 16 & Christofaro DGD et al & Educação Física & Ing & 2009 & Brasil & J. Pediatr. \\
\hline 17 & Fernandes RA et al & Educação Física & Ing & 2009 & Brasil & Arq Bras Cardiol \\
\hline 18 & Aglony $\mathrm{M}$ et al & Medicina & Esp & 2009 & Chile & Rev Méd Chile \\
\hline 19 & Costanzi CB et al & Fisioterapia & Ing & 2009 & Brasil & J. Pediatr. \\
\hline 20 & Salgado CM & Medicina & $\mathrm{Pt}$ & 2009 & Brasil & J. Pediatr. \\
\hline 21 & Zanoti MDU, Pina JC, Manetti ML & Enfermagem & $\mathrm{Pt}$ & 2009 & Brasil & Esc Anna Nery \\
\hline 22 & Pereira A et al & Medicina & Ing & 2009 & Brasil & Arq Bras Cardiol \\
\hline 23 & Sandoval R et al & Medicina & Esp & 2009 & Venezuela & Gac Méd Caracas \\
\hline 24 & Menezes AMB & Medicina & Ing & 2010 & Brasil & Cad. Saúde Pública \\
\hline 25 & lampolsky MN, Souza FIS, Sarni ROS & Medicina & $\mathrm{Pt}$ & 2010 & Brasil & Rev. Paul Pediatr \\
\hline 26 & Naghettini AV et al & Medicina & $\mathrm{Pt}$ & 2010 & Brasil & Sociedade Brasileira de Cardiologia \\
\hline 27 & Queiroz VM et al & Nutrição & $\mathrm{Pt}$ & 2010 & Brasil & Arq Bras Cardiol \\
\hline 28 & Szer G et al & Medicina & Esp & 2010 & Argentina & Arch Argent Pediatri \\
\hline 29 & Araújo FL et al & Educação Física & $\mathrm{Pt}$ & 2010 & Brasil & Rev.. Bras. Hipertens. \\
\hline 30 & Chaves ES et al & Enfermeira & $\mathrm{Pt}$ & 2010 & Brasil & Rev. Gaúcha Enferm. \\
\hline 31 & Díaz A & Medicina & Esp & 2010 & Argentina & Arch Argent Pediatr \\
\hline 32 & Ferreira JS, Aydos RD & Educação Física & $\mathrm{Pt}$ & 2010 & Brasil & Ciênc. Saúde coletiva \\
\hline 33 & Christofaro DGD et al & Educação Física & Ing & 2011 & Argentina & Arq Bras Cardiol \\
\hline 34 & Durrani AM, Fatima W & Nutrição & Ing & 2011 & Índia & European Journal of Public Health \\
\hline 35 & Mazaro IAR et al & Medicina & $\mathrm{Pt}$ & 2011 & Brasil & Rev. Assoc Med Bras \\
\hline 36 & Bancalari R et al & Medicina & Esp & 2011 & Chile & Rev. Med Chile \\
\hline 37 & Chehuen MR et al & Educação física & Ing & 2011 & Brasil & Rev. Bras Med Esporte \\
\hline 38 & Guo X et al & Não identificado & Ing & 2011 & China & Clinical Investigations \\
\hline 39 & Pinto SL et al & Nutrição & Ing & 2011 & Brasil & Cad. Saúde Pública \\
\hline 40 & Costa JV et al & Enfermagem & Ing & 2012 & Brasil & Rev. Latino-Am. Enfermagem \\
\hline 41 & Clemente APG et al & Nutrição & Ing & 2012 & Brasil & Arq Bras Cardiol \\
\hline 42 & Moselakgomo VK et al & Não identificado & $\mathrm{Pt}$ & 2012 & África do Sul & Rev. paul. Pediatr. \\
\hline 43 & Noronha JAF et al & Enfermagem & $\mathrm{Pt}$ & 2012 & Brasil & J. Hum. Growth Dev. \\
\hline 44 & reuter EM et al & Fisioterapia & Ing & 2012 & Brasil & Rev. Assoc. Med. Bras. \\
\hline 45 & Campagnolo PDB et al & Nutrição & $\mathrm{Pt}$ & 2013 & Brasil & Rev. Ciênc. Méd \\
\hline 46 & Moreira NF et al & Nutrição & $\mathrm{Pt}$ & 2013 & Brasil & Arq Bras Endocrinol Metab. \\
\hline 47 & Blanco LU et al & Medicina & Esp & 2013 & Chile & Salud Uninorte \\
\hline 48 & Ferreira CEF, Faria RJ, Bazoni HÁ & Medicina & $\mathrm{Pt}$ & 2013 & Brasil & Rev. Bras Cardiol. \\
\hline 49 & Milián RL et al & Medicina & Esp & 2013 & Cuba & Rev. Cubana Pediatr \\
\hline 50 & Mosera DC et al & Educação física & Ing & 2013 & Brasil & J. Pediatr. \\
\hline 51 & Fuly JTB et al & Medicina & Ing & 2014 & Brasil & Pediatr (Rio J). \\
\hline 52 & Kelishadia $\mathrm{R}$ et al & Não identificado & $\mathrm{Pt}$ & 2014 & Irã & J. Pediatr. \\
\hline 53 & Ladoux DB et al & Medicina & Esp & 2014 & Paraguai & Pediatr (Assunción) \\
\hline 54 & Moraes LI et al & Medicina & $\mathrm{Pt}$ & 2014 & Brasil & Arq Bras Cardiol \\
\hline
\end{tabular}

Fonte: dados da pesquisa. 
Quadro 2. Características gerais segundo delineamento, amostra, faixa etária, $\mathrm{n}^{\circ}$ de aferições, visitas, tempo de repouso e intervalo (A/V/R/I), manual, desfecho dos estudos sobre hipertensão em crianças e adolescentes publicados entre 2004 e 2014. Salvador, BA. 2015.

\begin{tabular}{|c|c|c|c|c|c|c|}
\hline $\mathbf{N}^{\circ}$ & Delineamento & Amostra & $\begin{array}{l}\text { Faixa } \\
\text { etária }\end{array}$ & $A / V / R / I$ & Manual & $\begin{array}{l}\text { Resultados/Desfecho/Prevalência da } \\
\text { PA (\%) }\end{array}$ \\
\hline 1 & Caso controle & 43 & 11 a 18 & $2 / 1 / 5 / 1$ & $\begin{array}{c}\text { III Consenso Brasileiro/ } \\
\text { Second Task Force on } \\
\text { Blood Pressure Control in } \\
\text { Children (1987) }\end{array}$ & $\begin{array}{l}\text { Os filhos dos hipertensos mostraram } \\
\text { maiores valores basais de pressão } \\
\text { arterial sistólica e diastólica }\end{array}$ \\
\hline 2 & Transversal & 672 & 2 a 10 & $2 / 1 / 5 / 5$ & $\begin{array}{c}\text { Task Force Report on } \\
\text { High Blood Pressure in } \\
\text { Children and Adolescents } \\
\text { (1987) }\end{array}$ & $\begin{array}{c}\text { Níveis mais elevados de pressão } \\
\text { arterial sistólica e diastólica } \\
\text { estiveram associados com crianças } \\
\text { de cor branca, crianças da região } \\
\text { com alto índice de qualidade de } \\
\text { vida urbana e com elevado índice de } \\
\text { massa corporal. }\end{array}$ \\
\hline 3 & Transversal & 701 & 5 a 9 & $2 / 1 / 2 / 2$ & $\begin{array}{c}\text { Task Force Report on } \\
\text { High Blood Pressure in } \\
\text { Children and Adolescents } \\
\text { (1987) }\end{array}$ & 3,6 \\
\hline 4 & Transversal & 1.253 & 7 a 17 & $2 / 1 / 2 / 2$ & $\begin{array}{c}\text { III Consenso Brasileiro de } \\
\text { Hipertensão Arterial }\end{array}$ & 7,7 \\
\hline 5 & Transversal & 58 & 10 a 19 & $3 / 3 / 0 / 0$ & $\begin{array}{c}\text { Task Force Report on } \\
\text { High Blood Pressure in } \\
\text { Children and Adolescents } \\
\text { (1987) }\end{array}$ & 65 \\
\hline 6 & Transversal & 1.253 & 7 a 17 & $2 / 1 / 2 / 1$ & $\begin{array}{c}\text { III Consenso Brasileiro } \\
\text { de Hipertensão } \\
\text { Arterial e do Update } \\
\text { on the } 1987 \text { Task Force } \\
\text { Report on High Blood } \\
\text { Pressure in Children and } \\
\text { Adolescents, resumidas } \\
\text { por Koch }\end{array}$ & 7,7 \\
\hline 7 & Transversal & 3749 & 12 a 16 & $4 / 2 / 10 / 5$ & $4^{\circ}$ Relatório (2004) & 2,6 \\
\hline 8 & Transversal & 456 & 12 a 17 & $6 / 3 / 10 / 5$ & IV DBHA & 4,6 \\
\hline 9 & Transversal & 150 & 7 a 17 & $3 / 3 / 10 / 10$ & Fourth Report (2004) & 40 \\
\hline 10 & Transversal & 3169 & 7 a 14 & $2 / 1 / 2 / 1$ & $\begin{array}{c}\text { Task Force Report on } \\
\text { High Blood Pressure in } \\
\text { Children and Adolescents } \\
\text { (1987) }\end{array}$ & 5,0 \\
\hline 11 & Transversal & 456 & 12 e 17 & $6 / 2 / 1 / 1$ & $\begin{array}{l}\text { IVDBHA e o Fourth } \\
\text { Report (2004) }\end{array}$ & 4,6 \\
\hline 12 & Transversal & 601 & 7 e 10 & $3 / 1 / 10 / 10$ & Fourth Report (2004) & 5,5 \\
\hline 13 & Transversal & 7.440 & 7 a 10 & $3 / 1 / 5 / 0$ & $\begin{array}{c}\text { Task Force Report on } \\
\text { High Blood Pressure in } \\
\text { Children and Adolescents } \\
\text { (1987) }\end{array}$ & 15 \\
\hline 14 & Transversal & 342 & 6 a 18 & $2 / 1 / 5 / 5$ & $\mathrm{AHA}$ & 44,7 \\
\hline 15 & Transversal & 1570 & 7 a 12 & $2 / 1 / 5 / 5$ & Fourth Report (2004) & $\begin{array}{l}\text { A razão de prevalência (RP) para o } \\
\text { excesso de peso associou-se às PAS e } \\
\text { PAD elevadas. }\end{array}$ \\
\hline 16 & Transversal & 1.021 & 10 a 17 & $2 / 1 / 5 / 2$ & I DAIA & $\begin{array}{c}\text { Valores da pressão arterial se } \\
\text { relacionaram significativamente com } \\
\text { O IMC }\end{array}$ \\
\hline 1 & Transversal & 1.145 & 11 a 17 & $2 / 1 / 5 / 2$ & I DAIA & 11,4 \\
\hline 18 & Coorte & 112 & 6 a 13 & $3 / 1 / 10 / 5$ & Fourth Report (2004) & 6,3 \\
\hline 19 & Transversal & 1.413 & 7 a 12 & $3 / 1 / 10 / 2$ & Fourth Report (2004) & 13,8 \\
\hline 20 & Transversal & 1.049 & 8 a 11 & $63 / 1 / 20 / 30$ & $\mathrm{AHA}$ & 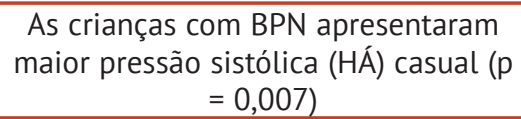 \\
\hline 21 & Transversal & 148 & 6 a 11 & $3 / 1 / 5 / 2$ & Fourth Report (2004) & 9,5 \\
\hline 22 & Transversal & 494 & 2 a 19 & $3 / 1 / 5 / 0$ & Fourth Report (2004) & 11,7 \\
\hline 23 & Transversal & 337 & 6 a 18 & $3 / 3 / 5 / 2$ & \begin{tabular}{|c|} 
Report of Second Task \\
Force on Blood Pressure \\
Control in Children-1987
\end{tabular} & 2,5 \\
\hline
\end{tabular}


Quadro 2. Continuação

\begin{tabular}{|c|c|c|c|c|c|c|}
\hline $\mathbf{N}^{\circ}$ & Delineamento & Amostra & $\begin{array}{l}\text { Faixa } \\
\text { etária }\end{array}$ & A/V/R/I & Manual & $\begin{array}{c}\text { Resultados/Desfecho/Prevalência da } \\
\text { PA (\%) }\end{array}$ \\
\hline 24 & Transversal & 4.452 & 1 a 11 & $2 / 1 / 1 / 1$ & Fourth Report (2004) & 7,1 \\
\hline 25 & Transversal & 1408 & 5 a 10 & $1 / 1 / 0 / 0$ & NHBPEP & 31 \\
\hline 26 & Transversal & 519 & 3 a 10 & $4 / 2 / 5 / 5$ & Fourth Report (2004). & 1,7 \\
\hline 27 & Transversal & 750 & 6 a 9 & $3 / 1 / 2 / 2$ & V DBHA & 13,6 \\
\hline 28 & Transversal & 816 & 6 a 9 & $3 / 1 / 0 / 0$ & NSAHA & 9,4 \\
\hline 29 & Transversal & 493 & 7 a 17 & $2 / 1 / 5 / 3$ & IV DBHA & 21 \\
\hline 30 & $\begin{array}{c}\text { Estudo } \\
\text { longitudinal/ } \\
\text { prospectivo }\end{array}$ & 141 & 6 a 18 & $2 / 1 / 30 / 30$ & V DBHA & 16,3 \\
\hline 31 & Transversal & 331 & 5 a 18 & $2 / 1 / 0 / 0$ & Fourth Report (2004). & 2,4 \\
\hline 32 & Transversal & 129 & 7 a 14 & $4 / 2 / 5 / 2$ & IV DBHA & 21 \\
\hline 33 & Transversal & 1.021 & 10 a 17 & $2 / 1 / 5 / 2$ & Fourth Report (2004) & 11,8 \\
\hline 34 & Transversal & 701 & 12 e 16 & $3 / 1 / 5 / 5$ & $\begin{array}{l}\text { Task Force on Blood } \\
\text { Pressure Control in } \\
\text { Children (1987) } \\
\end{array}$ & 9,4 \\
\hline 35 & Transversal & 680 & 7 e 11 & $2 / 2 / 2 / 3$ & Fourth Report (2004). & 10,9 \\
\hline 36 & Transversal & 2.980 & 6 a 14 & $3 / 1 / 5 / 5$ & Fourth Report (2004). & 13,6 \\
\hline 37 & Transversal & 205 & 7 a 18 & $3 / 1 / 5 / 5$ & V DBHA & 10,7 \\
\hline 38 & Transversal & 4902 & 5 e 18 & $2 / 1 / 5 / 2$ & Fourth Report (2004) & 17,65 \\
\hline 39 & Transversal & 1.125 & 7 e 14 & $2 / 1 / 10 / 10$ & V DBHA & 14,1 \\
\hline 40 & Transversal & 145 & 12 e 18 & $3 / 1 / 5 / 10$ & Fourth Report (2004) & 52,4 \\
\hline 41 & Transversal & 309 & 9 a 19 & $3 / 1 / 10 / 5$ & Fourth Report (2004) & $\begin{array}{c}\text { Valores Indivíduos com baixa estatura } \\
\text { leve e sobrepeso apresentaram } \\
\text { valores mais elevados da PAD }\end{array}$ \\
\hline 42 & Transversal & 1.172 & 10 a 16 & $3 / 1 / 5 / 5$ & $\begin{array}{l}\text { Report of the second task } \\
\text { force on blood pressure } \\
\text { control in children. } \\
\text { Pediatrics } 1987 \\
\end{array}$ & 3,5 \\
\hline 43 & Transversal & 200 & 2 a 18 & $3 / 1 / 5 / 1$ & V DBHA & 70,5 \\
\hline 44 & Transversal & 414 & 7 a 17 & $2 / 1 / 5 / 1$ & VI DBHA & 7,0 \\
\hline 45 & Transversal & 1.014 & 10 a 16 & $2 / 1 / 0 / 10$ & Fourth Report (2004) & 13,4 \\
\hline 46 & Transversal & 1.716 & 10 a 16 & $3 / 1 / 0 / 1$ & VI DBHA & 11,7 \\
\hline 47 & Transversal & 109 & 9 a16 & $2 / 2 / 2 / 2$ & Fourth Report (2004) & 19,26 \\
\hline 48 & Transversal & 1.342 & 7 a 14 & $3 / 3 / 5 / 1$ & VI DBHA & 1,5 \\
\hline 49 & Transversal & 100 & 4 a 18 & $3 / 3 / 3 / 3$ & Fourth Report (2004) & 9 \\
\hline 50 & Transversal & 1.441 & 10 a 16 & $3 / 1 / 5 / 2$ & V DBHA & 17.3 \\
\hline 51 & Transversal & 794 & 6 a 13 & $3 / 1 / 5 / 3$ & Fourth Report (2004) & 7 \\
\hline 52 & Transversal & 5680 & 10 a 18 & $2 / 1 / 5 / 2$ & Fourth Report (2004) & 6,25 \\
\hline 53 & Transversal & 287 & 5 a 18 & $3 / 3 / 5 / 5$ & Fourth Report (2004) & 17,7 \\
\hline 54 & Transversal & 800 & 9 a16 & $3 / 1 / 5 / 3$ & Fourth Report (2004) & 3,9 \\
\hline
\end{tabular}

Fonte: dados da pesquisa.

apresentaram $23(47,9 \%)$ produções com variação de prevalência ente $1,5 \%$ e $10 \%$; 17 (35,4\%) entre $11 \%$ e $20 \%$; $5(10,4 \%)$ entre $21 \%$ e $49 \%$ e; $3(6,35 \%)$ entre $50 \%$ e $75 \%$. Outras pesquisas também demonstraram variações na prevalência entre $3,5 \%$ e $50,7 \%$, 15 , tal característica pode estar relacionada, diretamente, aos métodos utilizados pelos pesquisadores, diferentes faixas etárias e ponto de cortes designados para definir a pressão elevada.

0 quadro 3 revela as características essenciais dos manuais utilizados nos artigos selecionados para este estudo descrevendo o ano de publicação, visitas e números de aferições recomendadas, tempo de repouso antes da aferição e nos intervalos entre elas, braço recomendado para aferição, bem como seus pontos de corte.
Neste estudo foram identificados 10 manuais que serviram de base para os estudos sobre prevalência da hipertensão arterial em crianças e adolescentes:

Report of the Second Task Force on Blood Pressure Control in Children (1987): trata-se da versão revisada do relatório original Task Force (1977), incluindo os dados relativos à amostragem de mais de 70.000 crianças, classificação dos níveis de PA, conselhos sobre a metodologia e instrumentação para a medição da PA, diagnóstico, avaliação e tratamentos farmacológico e não farmacológico na infância e adolescência ${ }^{16}$.

Update on the 1987 Task Force Report on High Blood Pressure in Children and Adolescents: A Working Group Report from The National High Blood Pressure Education 
Quadro 3. Informações sobre os manuais segundo ano de publicação, aparelho recomendado, número de visitas e aferições, Tempo (min) de repouso e intervalo entre as aferições, braço recomendado e ponto de corte dos estudos sobre hipertensão em crianças e adolescentes, publicados entre 2004 e 2014. Salvador, BA. 2015.

\begin{tabular}{|c|c|c|c|c|c|c|}
\hline $\begin{array}{l}\text { Manual/ Ano de } \\
\text { publicação }\end{array}$ & $\begin{array}{c}\text { Aparelho } \\
\text { recomendado } \\
\text { (Esfigmomanômetro) }\end{array}$ & $\begin{array}{c}\text { Número de } \\
\text { visitas }\end{array}$ & $\begin{array}{l}\text { Número de } \\
\text { aferições } \\
\text { recomendadas } \\
\text { em cada visita }\end{array}$ & $\begin{array}{c}\text { Repouso } \\
\text { (min) antes } \\
\text { da aferição/ } \\
\text { Intervalo (min) } \\
\text { entre aferições }\end{array}$ & $\begin{array}{c}\text { Braço } \\
\text { recomendado }\end{array}$ & $\begin{array}{c}\text { Ponto de corte } \\
\text { (mmHg; Percentil/ } \\
\text { idade e sexo) }\end{array}$ \\
\hline $\begin{array}{l}\text { Report of the Second } \\
\text { Task Force on Blood } \\
\text { Pressure Control in } \\
\text { Children-1987/ } 1987\end{array}$ & Coluna de mercúrio & ----- & ----- & -----/---- & MSD & $\begin{array}{l}\text { PA Normal: < } 90 . \\
\text { PA Normal alta: } \\
\text { entre } \\
90 \text { e } 95 . \\
\text { Hipertensão: entre } \\
95 \text { e } 99 . \\
\text { Hipertensão } \\
\text { severa: } \geqslant 99 .\end{array}$ \\
\hline $\begin{array}{l}\text { Update on the } 1987 \\
\text { Task Force Report } \\
\text { on High Blood } \\
\text { Pressure in Children } \\
\text { and Adolescents: } \\
\text { A Working Group } \\
\text { Report from The } \\
\text { National High Blood } \\
\text { Pressure Education } \\
\text { Program/1986 }\end{array}$ & Aneroide & $\geqslant 3$ & $\geqslant 2$ & 3 a 5/------ & MSD & $\begin{array}{l}\text { PA Normal: <90. } \\
\text { PA Normal alta: } \\
\text { entre } 90 \text { e } 95 . \\
\text { Hipertensão: entre } \\
95 \text { e } 99 . \\
\text { Hipertensão } \\
\text { severa: } \geqslant 99\end{array}$ \\
\hline $\begin{array}{l}\text { III Consenso } \\
\text { Brasileiro de } \\
\text { HAS/1998 }\end{array}$ & Coluna de mercúrio & $\geqslant 2$ & $\geqslant 2$ & 5 a $10 / 1$ a 2 & ----- & $\begin{array}{c}\text { Normal: }<90 \\
\text { Limítrofe } 90 \text { a } 95 \\
\text { HÁ }>95\end{array}$ \\
\hline IV DBHA/2004 & Coluna de mercúrio & $\begin{array}{c}\geqslant 2 \text { Se PAD } \\
\text { apresentar } \\
\text { diferenças } \\
>5 \mathrm{mHg} \\
\text { fazer novas } \\
\text { medidas }\end{array}$ & 2 & 5 a 10/----- & $\begin{array}{l}\text { MSD e E. . } \\
\text { Em caso de } \\
\text { diferença, } \\
\text { utilizar sempre } \\
\text { o braço de } \\
\text { maior pressão. }\end{array}$ & $\begin{array}{c}\text { Estágio } 1 \\
\text { PAS }=140-159 \\
P A D=90-99 \\
\text { Estágio } 2 \\
\text { PAS }=160-179 \\
\text { PAD }=100-109 \\
\text { Estágio } 3 \\
P A S=>180 \\
P A D=>110\end{array}$ \\
\hline $\begin{array}{l}\text { Fourth Report } \\
(2004) / 2002\end{array}$ & $\begin{array}{l}\text { Aneroide, } \\
\text { semiautomático } \\
\text { validado }\end{array}$ & $\begin{array}{c}\text { Múltiplas } \\
\text { medidas } \\
\text { de PA ao } \\
\text { longo de } \\
\text { semanas a } \\
\text { meses }\end{array}$ & $\geqslant 3$ & 5/----- & MSD & $\begin{array}{l}\text { Pré-hipertensão: } \\
\text { Entre os percentis } \\
90 \text { e } 95 . \text { Em } \\
\text { adolescentes, } \\
\text { se a PA } \geqslant \\
120 \times 80 \mathrm{mmHg} \\
\text { (mesmo se o } \\
\text { percentil < 90) } \\
\text { Hipertensão: } \\
\text { percentil } \geqslant 95 .\end{array}$ \\
\hline $\mathrm{AHA} / 2005$ & Coluna de mercúrio & ----- & $\geqslant 2$ & 3 a $5 / 1$ & MSD & $\geqslant$ percentil 95 \\
\hline I DPAIA/2005 & Coluna de mercúrio & ----- & ----- & -----/----- & MSD & $\begin{array}{c}\text { Normal: percentis } \\
\quad<90 \text {. } \\
\text { Pré-hipertensão: } \\
\text { percentis > } 90 \\
\text { e < } 95 \text { ou PA > } \\
\text { 120/80mmHg. } \\
\text { HAS estágio 1: } \\
\text { percentis entre } 95 \\
\text { e } 99 \\
\text { HAS estágio 2: PA } \\
\text { > percentis } 99\end{array}$ \\
\hline
\end{tabular}


Quadro 3. Continuação

\begin{tabular}{|c|c|c|c|c|c|c|}
\hline $\begin{array}{l}\text { Manual/ Ano de } \\
\text { publicação }\end{array}$ & $\begin{array}{c}\text { Aparelho } \\
\text { recomendado } \\
\text { (Esfigmomanômetro) }\end{array}$ & $\begin{array}{l}\text { Número de } \\
\text { visitas }\end{array}$ & $\begin{array}{l}\text { Número de } \\
\text { aferições } \\
\text { recomendadas } \\
\text { em cada visita }\end{array}$ & $\begin{array}{c}\text { Repouso } \\
\text { (min) antes } \\
\text { da aferição/ } \\
\text { Intervalo (min) } \\
\text { entre aferições }\end{array}$ & $\begin{array}{c}\text { Braço } \\
\text { recomendado }\end{array}$ & $\begin{array}{c}\text { Ponto de corte } \\
\text { (mmHg; Percentil/ } \\
\text { idade e sexo) }\end{array}$ \\
\hline V DBHA/2006 & $\begin{array}{c}\text { Coluna de mercúrio ou } \\
\text { aneroide }\end{array}$ & 3 & $\begin{array}{c}3 \\
\text { (considerando } \\
\text { a média das } \\
\text { duas últimas) }\end{array}$ & $5 / 1$ & $\begin{array}{c}\text { MSD e E. } \\
\text { Em caso de } \\
\text { diferença, } \\
\text { utilizar sempre } \\
\text { o braço de } \\
\text { maior pressão. }\end{array}$ & $\begin{array}{c}\text { Normal: PA }<130 \\
<85 \\
\text { Limítrofe: PA 130- } \\
13985-89 \\
\text { Estágio 1: PA } 140- \\
159 \text { 90-99 } \\
\text { Estágio 2: PA } 160- \\
179 \text { 100-109 } \\
\text { Estágio 3: PA } \geqslant \\
180 \geqslant 110 \\
\text { Hipertensão } \\
\text { sistólica isolada: } \geqslant \\
140<90\end{array}$ \\
\hline VI DBHA/2010 & $\begin{array}{c}\text { Aneroide ou } \\
\text { automáticos validados }\end{array}$ & $\geqslant 3$ & 2 & $5 / 1$ & $\begin{array}{c}\text { MSD e E. } \\
\text { Em caso de } \\
\text { diferença, } \\
\text { utilizar sempre } \\
\text { o braço de } \\
\text { maior pressão. }\end{array}$ & $\begin{array}{c}\text { Normal: PA < } \\
\text { percentil } 90 \\
\text { Limítrofe: PA entre } \\
\text { percentis de } 90 \text { a } \\
95 \text { ou se PA exceder } \\
120 / 80 \text { mmHg } \\
\text { sempre < percentil } \\
90 \text { até < percentil } \\
95 \\
\text { Hipertensão estágio } \\
\text { 1: Percentil } 95 \text { a } 99 \\
\text { mais } 5 \text { mmHg } \\
\text { Hipertensão estágio } \\
\text { 2: PA > percentil } 99 \\
\text { mais } 5 \text { mmHg } \\
\text { Hipertensão do } \\
\text { avental branco: } \\
\text { PA > percentil } 95 \\
\text { em ambulatório } \\
\text { ou consultório } \\
\text { e PA normal em } \\
\text { ambientes não } \\
\text { relacionados à } \\
\text { prática clínica }\end{array}$ \\
\hline NSAHA/NE & $\begin{array}{c}\text { Aneroide o } \\
\text { automáticos validados }\end{array}$ & $\geqslant 3$ & $\geqslant 2$ & $1 / 1$ & $\begin{array}{c}\text { MSD e E. } \\
\text { Em caso de } \\
\text { diferença, } \\
\text { utilizar sempre } \\
\text { o braço de } \\
\text { maior pressão. }\end{array}$ & $\begin{array}{l}\text { PA Normal: < } 90 . \\
\text { PA Normal alta: } \\
\text { entre } 90 \text { e } 95 . \\
\text { Hipertensão: entre } \\
95 \text { e } 99 . \\
\text { Hipertensão } \\
\text { severa: } \geqslant 99 .\end{array}$ \\
\hline
\end{tabular}

Fonte: dados da pesquisa.

Nota: $P A=$ pressão arterial MSD e $E=$ membro superior direito e esquerdo AHA = American Heart Association HAS= hipertensão arterial sistêmica PAS= pressão arterial sistólica $\mathrm{PAD}=$ pressão arterial diastólica $\mathrm{mmHg}=$ milímetro de mercúrio I DPAIA=Diretriz de Prevenção da Aterosclerose na Infância e na Adolescência NHBPEP= National High Blood Pressure Education Program Working Group on High Blood Pressure in Children and Adolescents NSAHA= Normas de la Sociedad Argentina de Hipertensión Arterial NE= não encontrado.

Program (1996): atualização do Relatório da Força Tarefa de 1987. Além de fornecer novas tabelas de Pas para crianças e adolescentes, que agora incluem percentis de altura, idade e sexo, estabelece o som de Korotkoff para definir PA diastólica e, discute-se a prevenção primária da hipertensão arterial nesses grupos etários ${ }^{17}$.
III Consenso Brasileiro de Hipertensão Arterial Sistêmica (1998): teve como objetivos revisar, atualizar e expandir o documento do II Consenso Brasileiro de Hipertensão Arterial, de 1994. O principal objetivo foi adequar à realidade da comunidade brasileira, apresentando como inovador a abordagem multiprofissional do hipertenso. 
Recomenda como parte integrante de sua avaliação clínica a determinação da pressão arterial à semelhança dos critérios já descritos para adultos e determinada a pressão diastólica na fase $\mathrm{V}$ de Korotkoff. Ainda, classifica como hipertensão arterial em crianças: valores abaixo do percentil 90, como normotenso; entre os percentis 90 e 95, normal limítrofe e; acima do percentil 95, hipertensão $\operatorname{arterial}^{18}$.

IV Diretrizes Brasileira de Hipertensão Arterial (2004): instituído para atualizar o III Consenso Brasileiro de Hipertensão Arterial, publicado em 1998. Neste foi empregado o grau de recomendação baseado no nível de evidência, de acordo com as normas da Associação Médica Brasileira. Foram acrescidos dois novos capítulos, os de Epidemiologia e Hipertensão Secundária. Apresenta a classificação de HA em crianças e adolescentes de acordo com os percentis de estatura e sexo, considerando-se hipertensão arterial valores iguais ou acima do percentil 95. Orienta pelo menos duas aferições em cada visita, repetindo-as caso apresente diferenças acima de $5 \mathrm{mmHg}$ entre elas, com medições em ambos os membros superiores e, recomenda-se que as medidas sejam repetidas em pelo menos duas ou mais visitas antes de confirmar o diagnóstico de hipertensão ${ }^{19}$.

The Fourth Report on the Diagnosis, Evaluation, and Treatment of High Blood Pressure in Children and Adolescents (2004): baseado em evidência científica, com o propósito de atualizar as últimas recomendações a respeito do diagnóstico, avaliação e tratamento da hipertensão em crianças. Orienta a aferição da PA em crianças a partir de três anos nas consultas médicas; inclui nas tabelas os percentis 50, 90, 95 e 99 por sexo, idade, altura. A PA elevada continua sendo classificada como percentil igual ou maior que 95 para sexo, idade e altura. No entanto, percentil entre 90 e 95 passa a ser chamado de "préhipertensão", indicando-se mudanças no estilo de vida. Também, assim como em adultos, adolescentes com níveis pressóricos iguais ou superiores a 120/80 mmHg devem ser considerados pré-hipertensos. Aconselha-se que as mensurações sejam realizadas no braço direito, repouso prévio de cinco minutos e que pelo menos as aferições sejam realizadas em três ou mais ocasiões diferentes ${ }^{20}$.

American Heart Association (2005): esta é a sexta edição da American Heart Association, traz recomendações para a medição indireta em crianças e adolescentes com base no Task Force on Blood Pressure Control in Children, dialoga sobre os tamanhos de manguitos pediátricos, sugere padronização de métodos na medição da PA no dia a dia dos cuidadores, em pesquisas epidemiológicas, educação em saúde e estudos clínicos ${ }^{21}$.

I Diretriz de Prevenção da Aterosclerose na Infância e na Adolescência (2005): trata-se de uma referência para o estabelecimento de estratégias individuais e populacionais no controle dos fatores de risco para aterosclerose na infância e adolescência. Mostra as principais causas de hipertensão arterial por faixa etária, recomenda pelo menos que seja realizada duas aferições, preferencialmente, no braço direito, em duas ocasiões distintas, com repouso de no mínimo 5 minutos, antes da obtenção da primeira medida 22 .

V Diretrizes Brasileira de Hipertensão Arterial (2006): apresentam mudanças importantes na prevenção, no diagnóstico, no tratamento e no controle da hipertensão arterial. Indica que em cada consulta haja o repouso prévio de pelo menos 5 minutos em ambiente calmo, realize pelo menos três medidas, com intervalo de um minuto entre elas, sendo a média das duas últimas considerada a pressão arterial do indivíduo. Classifica a pressão arterial para crianças e adolescentes como normal (PA < percentil 90), Limítrofe (PA entre percentis 90 a 95 ou se PA exceder 120/80 mmHg sempre < percentil 90 até < percentil 95), hipertensão estágio 1 (Percentil 95 a 99 mais $5 \mathrm{mmHg}$ ), hipertensão estágio 2 (PA > percentil 99 mais $5 \mathrm{mmHg}$ ), hipertensão do avental branco (PA > percentil 95 em ambulatório ou consultório e PA normal em ambientes não relacionados à prática clínica). Ressalta-se que o tratamento não medicamentoso deve ser recomendado a partir do percentil 90 de pressão arterial sistólica ou diastólica ${ }^{23}$.

VI Diretrizes Brasileira de Hipertensão Arterial (2010): resulta da necessidade de uma atualização de sua última versão de 2006, imposta pelo grande número de novas informações geradas e disponibilizadas nesse período de tempo. A medida da PA em crianças é recomendada em toda avaliação clínica, após os 3 anos de idade, pelo menos anualmente, como parte do seu atendimento pediátrico primário, devendo respeitar as padronizações estabelecidas para os adultos. Hipertensão arterial na infância é definida como pressão igual ou maior ao percentil 95 de distribuição da PA considerando a idade, o sexo e a altura. No caso de uma segunda medida consecutiva, sugere-se que haja um intervalo mínimo de um minuto ${ }^{24}$.

Normas de la Sociedad Argentina de Hipertensión Arterial (ne): neste recomenda-se que a aquisição da PA deva ser parte do exame pediátrico de rotina a partir dos primeiros dias de vida, considerando a história familiar de hipertensão. A hipertensão em crianças é definida de acordo com os percentis de PA por sexo, idade e altura, de acordo com o The Fourth eporto $\mathrm{n}$ the Diagnosis, Evaluation, and Treatment of High Blood Pressure in Children And Adolescents em 3 ou mais ocasiões ${ }^{25}$.

A despeito das semelhanças entre os manuais no que se refere à técnica de mensuração da hipertensão em crianças, observa-se que, algumas normatizações são necessárias, por exemplo, quanto ao número de ocasiões ou visitas mínimas e quantidade de aferições, como demonstrado no quadro 1, a média de prevalência, geralmente, é maior em estudos os quais se observa menor frequência de aferições e visitas, ou seja, a prevalência tem relação inversamente proporcional à quantidade de aferições realizadas ${ }^{14}$. Há uma superestimação nos valores reais da pressão arterial quando a medida acontece em apenas um dia e uma 
aferição, pois pesquisas apresentam resultados em que após avaliarem 6763 crianças em três ocasiões distintas, encontraram prevalência de PA de $11,4 \%$ no primeiro dia, $3,8 \%$ no segundo e, de $2,2 \%$ no terceiro ${ }^{4-15}$.

A tabela seguinte explicita a frequência da utilização dos manuais nos estudos analisados.

Tabela 1. Frequência da utilização dos manuais nos estudos analisados. Salvador, BA. 2015.

\begin{tabular}{c|c|c|c}
\hline Manuais & Estudos & $\mathbf{N}^{\circ}$ & $\%$ \\
\hline $\begin{array}{c}\text { Report of the Second Task } \\
\text { Force on Blood Pressure } \\
\text { Control in Children-1987 }\end{array}$ & $\begin{array}{c}2,3,5,10,13, \\
23,34,42\end{array}$ & 8 & 14,8 \\
\hline $\begin{array}{c}\text { Update on the 1987 Task } \\
\text { Force Report on High Blood } \\
\text { Pressure in Children and } \\
\text { Adolescents: A Working } \\
\text { Group Report from The } \\
\text { National High Blood } \\
\text { Pressure Education Program }\end{array}$ & 25 & 1 & 1,8 \\
\hline $\begin{array}{c}\text { III Consenso Brasileiro de } \\
\text { HAS }\end{array}$ & $1,4,6$ & 3 & 5,6 \\
\hline IV DBHA & $8,27,29,32$ & 4 & 7,4 \\
\hline Fourth Report (2004) & $\begin{array}{c}7,9,11,12,15,18, \\
19,21,22,24,26,\end{array}$ & & \\
\hline AHA & $40,41,45,36,38,47,49$, & 25 & 46,3 \\
\hline I DPAIA & $14,52,53,54$ & & \\
\hline V DBHA & 14,20 & 2 & 3,7 \\
\hline VI DBHA & $44,37,39,43,50$ & 5 & 9,3 \\
\hline NSAHA & 28 & 3 & 5,6 \\
\hline Total & & 1 & 1,8 \\
\hline & & 54 & 100 \\
\hline & & & 3,7 \\
\hline
\end{tabular}

Fonte: dados da pesquisa.

Nota: $\mathrm{AHA}=$ American Health Association DPAIA= Diretriz de Prevenção da Aterosclerose na Infância e na Adolescência DBHA= Diretrizes Brasileira de Hipertensão Arterial NSAHA= Guias de la sociedad argentina de hipertension para el diagnostico, estudio, tratamiento y seguimiento de la hipertension arterial.

Nota-se que o manual mais utilizado entre os artigos encontrados foram: o The Fourth Report on The Diagnosis, Evaluation, and Treatment of High Blood Pressure in Children and Adolescents, com 46,3\%; o Report of the Second Task Force on Blood Pressure Control in Children, 14,8\%; IV Diretrizes Brasileira de Hipertensão Arterial (7,4\%); V Diretrizes Brasileira de Hipertensão Arterial (9,3\%), o III Consenso e a VI Diretriz Brasileira de Hipertensão Arterial, ambas 5,6\%.

Em estudo semelhante ${ }^{15}$, o manual mais utilizado em pesquisas sobre hipertensão em crianças e adolescentes foi o "The Fourth Report (2004)". Este resultado pode estar associado ao fato deste documento ter sido elaborado com amostra de crianças e adolescentes americanos, além do que, suas diretrizes referentes ao ponto de corte para pressão arterial elevada, são utilizadas em outros manuais.

Vale ressaltar que, a pesar de $70,4 \%$ dos artigos que compõem esse estudo terem sido realizados no Brasil, a maioria deles utilizou o manual supracitado como referência para seus estudos, quando existem diretrizes mais atuais publicadas no país. Quanto a isso, as justificativas podem estar atreladas ao fato do manual americano estar a mais tempo disponível e os demais manuais o utilizarem como referência para classificarem a hipertensão arterial em crianças e adolescentes.

No entanto, vale a pena promover discussões a respeito das diferenças no que concerne à realidade das crianças e adolescentes americana e brasileira, como exemplo, a alimentação, que influencia diretamente nos níveis de PA, dentre outras questões.

Assim, pesquisas sobre a temática devem continuar sendo exploradas, principalmente pelo panorama crescente em que a prevalência de hipertensão arterial se apresenta na infância e adolescência em todo o mundo. Além disso, seria importante que arguições sobre a utilização de manuais que norteiam a identificação, o tratamento e o controle da doença incidam na perspectiva das especificidades infanto-juvenis de cada localidade.

\section{CONCLUSÃO}

A revisão integrativa possibilitou a construção de uma síntese do conhecimento científico acerca da prevalência de hipertensão arterial em crianças e adolescentes e os manuais utilizados nesses estudos, no período de 2004 a 2014.

Foi possível identificar 54 artigos para composição da análise. Nesta, constatou-se uma variação de prevalência de hipertensão arterial em crianças e adolescentes entre 1,5 e $70,5 \%$. O manual intitulado "The Fourth Report on The Diagnosis, Evaluation, and Treatment of High Blood Pressure in Children and Adolescents" foi utilizado com maior frequência entre as produções analisadas, apresentando uma abrangência de $46,3 \%$ dos artigos inclusos. Ademais, todas as produções elegíveis apontaram relação ou associação da prevalência com sobrepeso ou obesidade, bem como o sedentarismo e hábitos alimentares inapropriados.

Este estudo mostrou-se importante à medida que as informações podem ser utilizadas para conduzir investigações científicas, bem como as práticas de saúde dos profissionais de enfermagem, permitindo um avanço positivo na identificação precoce no que tange a hipertensão arterial em crianças e adolescentes. Ainda, pode nortear ações educativas para prevenção da hipertensão essencial em crianças e adolescentes, uma vez que denuncia alguns fatores de risco em que crianças e adolescentes estão expostos.

Desta forma, recomendamos um aprofundamento na temática quanto ao número de visitas mínimas e quantidade de aferições realizadas em cada ocasião, uma vez que tais aplicações têm mostrado significância no que se refere à variação da prevalência de hipertensão arterial na infância e adolescência. 


\section{REFERÊNCIAS}

1. Moura ADA, Mendonça MG, Lima GG, Farias LM, Feitosa AR, Chaves ES. Atuação do enfermeiro sob a ótica do usuário hipertenso. Rev Rene. 2012; 13(3):504-13.

2. Mansano NG, Vila VSC, Rossi LA. Conhecimentos e necessidades de aprendizagem relacionadas à enfermidade cardíaca para hipertensos revascularizados em reabilitação. Rev. Eletr. Enf. 2009;11(2):349-59.

3. Moreira JP, Moraes JR, Luiz RR. Prevalence of self-reported systemic arterial hypertension in urban and rural environments in Brazil: a population-based study. Cad. Saúde Pública. jan 2013; 29(1):62-72.

4. Bezerra MLO, Soares PFC, Leite ES, Lucena RCS. Hypertension in Children and Adolescents: a systematic review about prevalence and risk factors. Rev enferm UFPE on line. 2013; 7(8):5313-22.

5. Guo X, Zheng L, Li Y, Yu S Liu S, Zhou X, et al. Association Between Sleep Duration and Hypertension Among Chinese Children and Adolescents. Clin. Cardiol. 2011; 34(1): 774781.

6. Oliveira R, Lamounier JÁ, Oliveira ADB, Castro MDR.; Oliveira JS. Pressão arterial em escolares e adolescentes - 0 estudo de Belo Horizonte. Jornal de Pediatria. 1999; 75(4): 256-266.

7. Kohlmann NEB, Kohlmann OJ. Histórico e perspectivas da medida da pressão arterial. Revista Hipertensão. set/out 2011; 14(2):5-13.

8. Koch VH.Aspectos Diagnósticos e Terapêuticos Atuais da Hipertensão Arterial na Criança e no Adolescente, com Ênfase no "Fourth Report on the Diagnosis, Evaluation, and Treatment of High Blood Pressure in Children and Adolescents" de 2004.J Bras Nefrol. 2005; XXVII (2):84-92.

9. Bezerra SAG, Saenger AL, Azambuja RL, Brandão AA. Como tratamos hipertensão na criança e no adolescente. Rev Bras Cardiol. 2013;26(2):86-89.

10. Sociedade Brasileira de Cardiologia. VI Diretrizes Brasileiras de Hipertensão. Arquivos Brasileiros de Cardiologia [Internet]. 2010:7-28. Disponível em: http://departamentos. cardiol.br/dha/vidiretriz/vidiretriz.asp. Acesso: 07 jun. 2013.

11. Bublitz S, Guido LA, Freitas EO, Lopes LFD. Estresse em estudantes de enfermagem: uma revisão integrativa. Rev Enferm UFSM. set/dez 2012;2(3):530-38.

12. Souza MT, Silva MD, Carvalho R. Integrative review: what is it? How to do it? Einstein. 2010; 8(1 Pt 1):102-6.

13. Paula CF, Andrade TCB. Atuação do enfermeiro na prevenção de hipertensão arterial e diabetes mellitus na família. 2012: 16 (1): p. 137- 48

14. OliveiraTMF, Almeida TCF. Adequacy of the cuff during blood pressure measurement: an integrative review. Ciência \& Saúde 2015;8(1):35-41.

15. Christofaro DGD, Andrade SM, Fernandes RA, Cabrera MAS, Ritti-Dias RM. Prevalência de pressão arterial elevada em crianças e adolescentes: revisão sistemática. Rev. Bras. Saúde Matern. Infant. 2011; 11 (4): 361-367.

16. Report of the Second Task Force on Blood Pressure Control in Children--1987. Task Force on Blood Pressure Control in Children. National Heart, Lung, and Blood Institute, Bethesda, Maryland. Pediatrics. 1987 Jan;79(1):1-25.
17. Update on the 1987 Task Force Report on High Blood Pressure in Children and Adolescents: A Working Group Report from The National High Blood Pressure Education Program. Pediatrics. 1996; 98(4):649-59.

18. III Consenso Brasileiro de Hipertensão Arterial. Arq Bras Endocrinol Metab. 1999; 43(4):257-86.

19. Sociedade Brasileira de Cardiologia.IV Diretrizes Brasileiras de Hipertensão Arterial [Internet]. 2004 [acesso jul 2015]. Disponível: http://publicacoes.cardiol.br/consenso/2004/ Diretriz\%20HA.pdf.

20. National High Blood Pressure Education Program Working Group on Hypertension Control in Children and Adolescents. The Fourth Report on the diagnosis, evaluation, and treatment of high blood pressure in children and adolescents. Pediatrics. 2004; 114: 555-76.

21. Pickering TG, Hall JE, Appel $\sqcup$, Falkner BE, Graves J, Hill MN, et al. Recommendations for blood pressure measurement in humans and experimental animals. Part 1: Blood pressure measurement in humans: as treatment for professionals from the subcommittee of professional and public education of the American Heart Association Council on High Blood Pressure Research. Circulation. 2005; 111: 697-716.

22. Giuliano IC, Caramelli B, Pellanda L, Duncan B, Mattos S, Fonseca $\mathrm{FH}$, editores. I Diretriz de Prevenção da Aterosclerose na Infância e na Adolescência. Arq Bras Cardiol. 2005;85 Supl 6:3-36.

23. Sociedade Brasileira de Cardiologia. V Diretrizes Brasileiras de Hipertensão Arterial. Arq Bras Cardiol. 2007; 89(3):24-79.

24. Sociedade Brasileira de Cardiologia, Sociedade Brasileira de Hipertensão, Sociedade Brasileira de Nefrologia. VI Diretrizes Brasileiras de Hipertensão. Arq Bras Cardiol. 2010; 95 (1 supl.1):1-51.

25. Sociedad Argentina de Cardiología. Guias de la sociedad argentina de hipertension para el diagnostico, estudio, tratamiento y seguimiento de la hipertension arterial [Internet]. (sd): 1-113. Disponível em: http://www.saha.org. ar/1/pdf/GUIA_SAHA_VERSION_COMPLETA.pdf. Acesso: 16 fev. 2015. 